\title{
On some definite integrals involving Legendre functions
}

\author{
By N. G. Shabde (University of Edinburgh).
}

(Received 5th December, 1933, and in revised form 30th January, 1934. Read 12th January, 1934.)

1. A few definite integrals involving more than two Legendre functions in the integrand have been considered by Ferrers, Adams, Dougall, Nicholson and Bailey. We take for example the following integrals.

$$
\begin{aligned}
& { }^{1}(1 \cdot 1) \int_{-1}^{1} P_{p}(\mu) P_{q}(\mu) P_{r}(\mu) d \mu, \\
& { }^{2}(1 \cdot 2) \int_{-1}^{1}\left(1-\mu^{2}\right)^{\frac{1}{m}} P_{p}^{m}(\mu) P_{q}^{m}(\mu) d \mu \text { or } \\
& { }_{3}^{1}\left(1-\mu^{2}\right)^{3 m} P_{p}^{m}(\mu) P_{q}^{m}(\mu) d \mu, \\
& { }^{4}(1 \cdot 3) \int_{-1}^{1} P_{a}(\mu) P_{\beta}(\mu) Q_{\gamma}(\mu) d \mu \text { and } \\
& { }^{2}(1 \cdot 4) \int_{-1}^{1}\left\{P_{p}(\mu)\right\}^{4} d \mu .
\end{aligned}
$$

It will be interesting to consider other definite integrals of the above type with three Legendre functions in the integrand, various possible combinations of the Legendre functions of the two kinds being taken into account. We also generalize for unrestricted values of $m$ and $n$ the definite integrals

$$
\begin{aligned}
& \int_{-1}^{1} P_{n}(\mu) Q_{m}(\mu) d \mu, \\
& \int_{-1}^{1} Q_{n}(\mu) d \mu \text { and } \\
& \int_{0}^{1} P_{n}(\mu) Q_{m}(\mu) d \mu,
\end{aligned}
$$

considered by Nicholson ${ }^{5}$ for integer-values of $n$ and $m$ only.

1 Hobson, Spherical and Ellipsoidal Harmonics, p. 87. The references to Ferrers and Adams, who considered this integral, are given there.

2 W. N. Bailey, "On the product of two Legendre functions," Proc. Oamb. Phil. Soc. 29 (1933), 173 , results $(4 \cdot 1)$ and $(4 \cdot 2)$.

3 J. Dougall, Proc. Edin. Math. Soc. 37 (1919), 33.47, formula (3).

4 Nicholson, Phil. Mag. (6) 43 (1922), 783.

5 Phil. Mag. (6), 43 (1922), 1-29, "Zonal Harmonics of the second kind." 
2. In the expansion for $\left(\mu^{2}-1\right)^{\frac{1}{2} m} P_{p}^{m}(\mu) \cdot Q_{q}^{m}(\mu)$ given by W. N. Bailey ${ }^{1}$ we put $m=0$. We have the result

$$
P_{p}(\mu) Q_{q}(\mu)=\sum_{r=0}^{p} \frac{A_{r} A_{p-r} A_{q-p+r}}{A_{q+r}} \frac{2 q-2 p+4 r+1}{2 q-2 p+2 r+1} Q_{q+p+2 r}(\mu)
$$

${ }^{2}$ where $\frac{\left(\frac{1}{2}\right)_{8}}{(s+m) !}=A_{s}^{m}$, i.e., $\frac{\left(\frac{1}{2}\right)_{q-r}}{(q+m-r) !}=A_{q-r}^{m} ; A_{r, m}=\frac{\left(\frac{1}{2}-m\right)_{r}}{r !}$; $A_{s}=A_{s}^{0}=A_{s, 0} ; A_{-s}^{m}=\frac{(-)^{8}}{\left(\frac{1}{2}\right)_{s}(m-s) !} ; q \geqq p$ and $p$ and $q$ are positive integers or zero.

From the definition

$$
Q_{n}(\cos \theta)=\frac{1}{2}\left\{Q_{n}(\cos \theta+0 \cdot i)+Q_{n}(\cos \theta-0 \cdot i)\right\}
$$

we see, after putting $\mu=\cos \theta+0 \cdot i$ and $\mu=\cos \theta-0 \cdot i$ successively in $(2 \cdot 1)$, adding and dividing by 2 , that $(2 \cdot 1)$ is also valid for $\mu=\cos \theta$, for real values of $\theta$. We multiply both the sides of $(2 \cdot 1)$ by $Q_{n}(\mu)$, where $R(n)>0$, and integrate with respect to $\mu$ from $\mu=-1$ to $\mu=+1$, remembering the results

${ }^{3}(2 \cdot 2) \int_{-1}^{1} Q_{m}(\mu) Q_{n}(\mu) d \mu$

$$
=\frac{\left[\{\psi(n+1)-\psi(m+1)\}\{1+\cos m \pi \cos n \pi\}-\frac{\pi}{2} \sin (n-m) \pi\right]}{(m-n)(m+n+1)}
$$

$=B_{m, n}$ say, where $R(m)>0, R(n)>0$ and $m \neq n$, and

${ }^{4}(2 \cdot 3) \int_{-1}^{1}\left\{Q_{n}(\mu)\right\}^{2} d \mu=\frac{1}{2 n+1}\left[\begin{array}{c}\pi^{2} \\ 2\end{array}-\left(1+\cos ^{2} n \pi\right) \psi^{(2)}(n+1)\right]=B_{n, n}$ say,

where $R(n)>0, \psi(t+1)=\frac{d}{d t}\{\log \Gamma(t+1)\}$ and $\psi^{(2)}(z)=\frac{d^{2}}{d z^{2}}\{\log \Gamma(z)\}$

1 L.c., formula (5*4).

2 I have to thank a referee for suggesting this notation and other modifications in this paper.

${ }^{3}$ Ganesh Prasad, "On non-orthogonal systems of Legendre's functions," Proc. Benares Math. Soc., 12 (1930), 33-42.

"Shabde, "On some series and integrals involving Legendre functions," Bull. Calcutta Math. Soc., 25 (1933), 29. 
We have

$$
\begin{aligned}
\int_{-1}^{1} P_{p}(\mu) Q_{q}(\mu) & Q_{n}(\mu) d \mu \\
& =\sum_{r=0}^{p} \frac{A_{r} A_{p-r} A_{q-p+r}}{A_{q+r}} \frac{2 q-2 p+4 r+1}{2 q-2 p+2 r+1} B_{q-p+2 r, n}
\end{aligned}
$$

where $R(n)>0, q \geqq p$ and $p$ and $q$ are positive integers or zero. As a simple deduction from $(2 \cdot 4)$ we have

$(2 \cdot 5) \int_{-1}^{1} P_{p}(\mu) Q_{q}(\mu) Q_{n}(\mu) d \mu=0$, if $q-p+n$ is an odd integer.

To evaluate $\int_{0}^{1} P_{p}(\mu) Q_{q}(\mu) Q_{n}(\mu) d \mu$,

where $p$ and $q$ are positive integers or zero such that $q \geqq p$ and $R(n)>0$, we proceed in the same manner as for $(2 \cdot 4)$ using in place of $(2 \cdot 2)$ and $(2 \cdot 3)$ the results

$1(2 \cdot 6) \int_{0}^{1} Q_{m} Q_{n} d \mu$

$$
\begin{aligned}
=\frac{1}{(m-n)(m+n+1)}\left[\psi(n+1)-\psi(m+1)-\pi \cos \frac{m \pi}{2} \sin \frac{n \pi}{2} \frac{\Pi\left(\frac{n-1}{2}\right) \Pi\left(\frac{m}{2}\right)}{\Pi\left(\frac{m-1}{2}\right) \Pi\left(\frac{n}{2}\right)}\right. \\
\left.+\pi \cos \frac{n \pi}{2} \sin \frac{m \pi}{2} \frac{\Pi\left(\frac{m-1}{2}\right) \Pi\left(\frac{n}{2}\right)}{\Pi\left(\frac{n-1}{2}\right) \Pi\left(\frac{m}{2}\right)}\right]
\end{aligned}
$$

$=C_{m, n}$ say, where $m \neq n, R(m)>0$ and $R(n)>0$; and

$$
(2 \cdot 7) \int_{0}^{1}\left[Q_{n}(\mu)\right]^{2} d \mu=\frac{1}{2} \int_{-1}^{1}[Q(\mu)]^{2} d \mu=\frac{1}{2 n+1}\left[\frac{\pi}{4}-\psi^{(2)}(n+1)\right]
$$

$=C_{n, n}$ say, where $n$ is a positive integer.

We have, finally,

$$
\begin{aligned}
& \int_{0}^{1} P_{p}(\mu) Q_{q}(\mu) Q_{n}(\mu) d \mu \\
& \quad=\sum_{r=0}^{p} \frac{A_{r} A_{p-r} A_{q-p+r}}{A_{q+r}} \frac{2 q-2 p+4 r+1}{2 q-2 p+2 r+1} C_{q-p+2 r, n}
\end{aligned}
$$

where $R(n)>0, q \geqq p$, and $p$ and $q$ are positive integers or zero.

1Dhar and Shabde, "On the non-orthogonality of Legendre's functions," Bull. Calcutta Math. Soc., 24 (1932), 185. 
When $n$ is a positive integer $(2 \cdot 4)$ can be deduced from (2.8), since $\int_{-1}^{1} P_{p}(\mu) Q_{q}(\mu) Q_{n}(\mu) d \mu=\left\{1+(-1)^{p+q+n}\right\} \int_{0}^{1} P_{p}(\mu) Q_{q}(\mu) Q_{n}(\mu) d \mu$. To evaluate $\int_{1}^{\infty} P_{p}(\mu) Q_{q}(\mu) Q_{n}(\mu) d \mu$, where $R(n)>0, q \geqq p, p$ and $q$ being positive integers or zero, we use $(2 \cdot 1)$ with the results

${ }^{1}$ (i) $\int_{1}^{\infty} Q_{n}(\mu) Q_{m}(\mu) d \mu=\frac{\psi(m+1)-\psi(n+1)}{(m-n)(m+n+1)}=D_{m, n}$ say, where $R(m)>0, R(n)>0, m \neq n$, and

(ii) $\int_{1}^{\infty}\left[Q_{n}(\mu)\right]^{2} d \mu=\frac{1}{2 n+1} \psi^{(2)}(n+1)=D_{n, n}$ say, $n$ being a positive integer.

So we have

$$
\begin{aligned}
& \int_{1}^{\infty} P_{p}(\mu) Q_{q}(\mu) Q_{n}(\mu) d \mu \\
& \quad=\sum_{r=0}^{p} \frac{A_{r} A_{p-r}}{A_{q+r}} \frac{A_{q-p+r}}{2 q-2 p+2 r+1} D_{q-p+2 r, n}
\end{aligned}
$$

where $R(n)>0, q \geqq p, q$ and $p$ being positive integers or zero.

3. Taking Ferrers' definitions for $P_{n}^{m}(\mu)$ and $Q_{n}^{m}(\mu)$, it can be directly shown that

$$
\begin{aligned}
& (3 \cdot 1) \int_{-1}^{1} Q_{n}^{m}(\mu) P_{p}^{m}(\mu) d \mu \\
& =\frac{1}{(p-n)(p+n+1)}\left[\left\{P_{p}^{m}(\mu) \frac{d}{d \mu} Q_{n}^{m}(\mu)-Q_{n}^{m}(\mu) \frac{d}{d \mu} P_{p}^{m}(\mu)\right\}\left(1-\mu^{2}\right)\right]_{-1}^{+1} \\
& =0, \text { if } p+n \text { is even, } \\
& =\frac{2}{(p-n)(p+n+1)} \frac{(p+m) !}{(p-m) !}, \text { if } p+n \text { is odd, } p \geqq m .
\end{aligned}
$$

Converting the expansion ${ }^{2}$ for $\left(\mu^{2}-1\right)^{\frac{3}{m}} P_{p}^{m}(\mu) P_{q}^{m}(\mu)$ into Ferrers' notations we get

$$
\begin{aligned}
& \text { (3.2) }\left(1-\mu^{2}\right)^{\frac{a}{m} m} P_{p}^{m}(\mu) P_{q}^{m}(\mu) \\
& =(-1)^{m} \frac{(p+m) !(q+m) !}{2^{m}(p-m) !(q-m) !} \sum_{r=0}^{q+m} \frac{A_{r, m} A_{q-r}^{m} A_{p-r}^{m}}{A_{p+q+m-r}^{-m}} \\
& \frac{2 q+2 p+2 m-4 r+1}{2 q+2 p+2 m-2 r+1} P_{p+q+m-2 r}^{m}(\mu) .
\end{aligned}
$$

1 Ganesh Prasad, l.c., p. 40.

W. N. Bailey, l.c., formula (3·3). 
Multiplying the two sides of $(3 \cdot 2)$ by $Q_{n}^{m}(\mu)$, and integrating with respect to $\mu$ from $\mu=-1$ to $\mu=1$, we have, after using $(3 \cdot 1)$,

(3.3) $\int_{-1}^{1}\left(1-\mu^{2}\right)^{\frac{3}{m} m} P_{p}^{m}(\mu) P_{q}^{m}(\mu) Q_{n}^{m}(\mu) d \mu=0$, if $p+q+m+n$ is even, and

$$
\begin{aligned}
=(-1)^{m} & \frac{(p+m) !}{2^{m}(p-m) !(q+m) !}{ }^{q+m} \sum_{r=0}^{q+m} \frac{A_{r, m} A_{q-r}^{m} A_{p-r}^{m}}{A_{p+q+m-r}^{m}} \frac{2 q+2 p+2 m-4 r+1}{2 p+2 q+2 m-2 r+1} \\
& \left(\bar{p}+\overline{q+} \frac{2}{m-2 r-n)} \frac{(p+q+2 m-2 r) !}{(p+q+m-2 r+n+1)(p+q-2 r) !}\right.
\end{aligned}
$$

if $p+q+m+n$ is odd such that $p+q>0, p-q \geqq 2 m$ and $n<p-q-m$.

4. To evaluate $\int_{-1}^{1} P_{p}^{m}(\mu) P_{q}^{m}(\mu) P_{n}^{t}(\mu)\left(1-\mu^{2}\right)^{\frac{t m}{m}} d \mu$, we use (3.2) with the result ${ }^{1}$

$$
\begin{aligned}
& (4 \cdot 1) \int_{-1}^{1} P_{q}^{m}(x) P_{n}^{t}(x) d x=0 \text { if } q+n \text { is odd, } \\
& =(-1)^{k} \frac{2}{2 n+1} \frac{(n+t) !}{(n-m) !} \text {, if } n=q \text { and } m-t=2 k, \\
& =\frac{4(n+t) !}{(n-t) !}\left[\sum_{8=0}^{r, k}(-1)^{k+8}{ }^{k} C_{s}{ }^{r+k-s-1} C_{k-1}(2 n+2 k-4 s+1)\right. \\
& \frac{(q-t-2 s) !(m+q) !(2 q-2 s) !(q-s+k) !(q-r-s) !}{(q+m-2 s) !(q-m) !(q-s) !(q-r-s+k-1) !} \\
& \left.\frac{(2 q-2 k-2 r-2 s-1) !}{(2 q-2 r-2 s+1) !(2 q+2 k-2 s+1) !}\right] \text { where } n=q-2 r,
\end{aligned}
$$

$m-t=2 k$, and the summation is taken over all values of $s$ from 0 to $r$ if $r<k$, and all values of $s$ from 0 to $k$ if $r>k$.

Hence if $p \geqq m, q \geqq m, n \geqq t, p-q \geqq 2 m$ and $m-t=2 k, k$ being any positive integer, we have

(4.2) $\int_{-1}^{1}\left(1-\mu^{2}\right)^{\frac{1}{2} m} P_{p}^{m}(\mu) P_{q}^{m}(\mu) P_{n}^{t}(\mu) d \mu=0$ when $p+q+m+n$ is an odd integer, and

$$
\begin{gathered}
=(-1)^{m} \frac{(p+m) !(q+m) !}{\frac{(p+m}{2^{m}(p-m) !(q-m) !} \sum_{l=0}^{q+m} \frac{A_{l, m} A_{q-l}^{m} A_{p-l}^{m}}{A_{p+q+m-l}^{-m}} \frac{2 p+2 q+2 m-4 l+1}{2 p+2 q+2 m-2 l+1}} \\
\int_{-1}^{1} P_{n}^{t}(\mu) P_{p+q+m-2 l}^{m}(\mu) d \mu,
\end{gathered}
$$

${ }^{1}$ H. K. Sirkar, Proc. Edin. Math. Soc., 2, 1 (1927-29), 244, " On the evaluation of $\int_{-1}^{1} P_{n}^{m} P_{7}^{p} d x$ and $\int_{0}^{1} P_{n}^{m} P_{g}^{p} d x . "$ 
where $m \leqq n<p-q-m<p+q+m$,

$$
\begin{gathered}
=\frac{(-1)^{m}(p+m) !(q+m) !}{2^{m}(p-m) !(q-m) !} \sum_{l=0}^{q+m} \frac{A_{l, m} A_{q-l}^{m} A_{p-l}^{m}}{A_{p+q+m-l}^{-n}} \frac{2 p+2 q+2 m-4 l+1}{2 p+2 q+2 m-2 l+1} \\
\frac{4(n+t) !}{(n-t) !}\left[\sum_{s=0}^{r, k}(-1)^{k+s}{ }^{k} C_{s}{ }^{r+k-8-1} C_{k-1}(2 n+2 k-4 s+1) !\right. \\
\\
\frac{(p+q+m-2 l-t-2 s) !(p+q+2 m-2 l) !(2 p+2 q+2 m-4 l-2 s) !}{(p+q+2 m-2 l-2 s) !(p+q-2 l) !(p+q+m-2 l-s) !}
\end{gathered}
$$

$\left.\frac{(p+q+m-2 l-s+k) !(p+q+m-2 l-r-s) !(2 p+2 q+2 m-4 l+2 k-2 r-2 s-1) !}{(p+q+m-2 l-r-s+k-1) !(2 p+2 q+2 m-4 l-2 r-2 s+1) !(2 p+2 q+2 m-4 l+2 k-2 s+1) !}\right]$

where $p+q+m-2 l-2 r=n$, the summation within [ ] being taken over all values of $s$ from 0 to $r$ if $r<k$, and all values of $s$ from 0 to $k$ if $r>k$.

5. Following the method given by Ganesh Prasad ${ }^{1}$ for evaluating $\int_{-1} Q_{m}(\mu) Q_{n}(\mu) d \mu$ for unrestricted values of $m$ and $n$ such that $\boldsymbol{R}(\boldsymbol{m})>0$, and $R(n)>0$, the following results can be easily worked out:-

$$
\begin{aligned}
& \int_{-1}^{1} P_{n}(\mu) Q_{m}(\mu) d \mu \\
& =\frac{-1+\cos (m-n) \pi-\frac{2}{\pi} \sin n \pi \cos m \pi\{\psi(m+1)-\psi(n+1)\}}{(m-n)(m+n+1)},
\end{aligned}
$$

where $R(m)>0, R(n)>0$ and $m \neq n$.

$\int_{-1}^{1} Q_{n}(\mu) d \mu=-\frac{1-\cos n \pi}{n(n+\mathrm{I})}$, where $R(n)>0$,

$=\frac{1}{(m-n)(m+n+1)}\left[-1+\frac{\Pi\left(\frac{n}{2}\right) \Pi\left(\frac{m-1}{2}\right)}{\Pi\left(\frac{n-1}{2}\right) \Pi\left(\frac{m}{2}\right)} \cos \left(\frac{n-m}{2}\right) \pi\right]$,

where $R(m)>0, R(n)>0$ and $m \neq n$.

These results reduce to the values given by Nicholson ${ }^{2}$, when $m$ and $n$ are positive integers.

1 L.c., p. 38.

2 Phil. Mag. (6), 43 (1922), 1-29. 